\title{
Cartografía de la revista Profesional de la información: mapa visual de 30 años de historia
}

\section{A cartography of the Profesional de la información journal: a visual map of $\mathbf{3 0}$ years of history}

\author{
Audilio Gonzales; María-Jesús Colmenero-Ruiz; Adilson-Luiz Pinto
}

Cómo citar este artículo:

Gonzales, Audilio; Colmenero-Ruiz, María-Jesús; Pinto, Adilson-Luiz (2021). "Cartografía de la revista Profesional de la información: mapa visual de 30 años de historia". Profesional de la información, v. 30, n. 6, e300605.

https://doi.org/10.3145/epi.2021.nov.05

Artículo recibido el $28-08-2021$

Aceptación definitiva: 25-10-2021

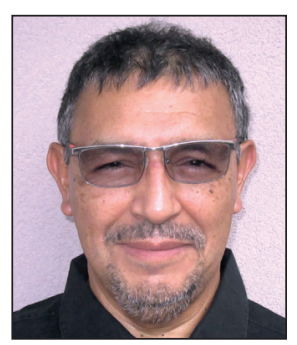

\section{Audilio Gonzales \\ https://orcid.org/0000-0001-8693-2076}

Université Montpellier 3

Route de Mende

34090 Montpellier, Francia

audilio.gonzales@univ-montp3.fr

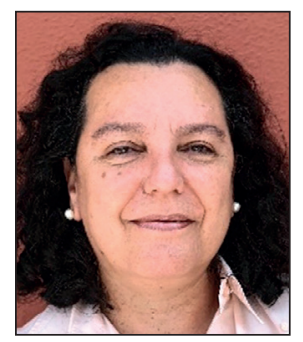

María-Jesús Colmenero-Ruiz

https://orcid.org/0000-0002-1650-1091

Universidad Complutense de Madrid

Facultad de Ciencias de la Documentación

Santísima Trinidad, 37

28010 Madrid, España

mcolmene@ucm.es

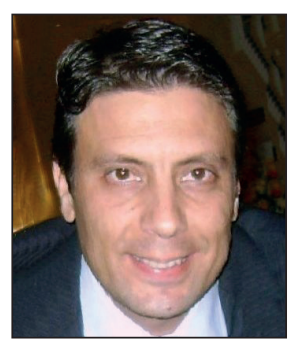

Adilson-Luiz Pinto

https://orcid.org/0000-0002-4142-2061

Universidade Federal de Santa Catarina

Campus Universitário - Trindade

88040-900 Florianópolis

Santa Catarina, Brasil

adilson.pinto@ufsc.br

\section{Resumen}

Se presenta el proceso de realización de una cartografía, una visualización interactiva, a partir de los datos bibliográficos de los contenidos de la revista científica Profesional de la Información en sus casi 30 años de vida. La cartografía se puede consultar en el sitio web de la revista EPI: https://profesionaldelainformacion.com/cartografia/EPI_GLOBAL/index.html. Su objetivo es servir como índice navegable para la localización rápida de información de interés para los lectores, así como un mapa visual que resalta las características de la red de conocimiento que esos datos forman a través de la interconexión de relaciones entre sus nodos. Este dispositivo ha sido realizado a partir de la extracción de datos de la web de la propia revista mediante programas de web scraping, y el software libre Gephi para la visualización de las redes. Un visor de gráficos permite el acceso y su manipulación a través de la web. La navegación por la red de datos bibliográficos se facilita mediante distintas estrategias: (1) la diferenciación por colores de cada categoría de nodo (autor, palabra clave, número y artículo); (2) las herramientas de búsqueda del visor: un buscador de etiquetas de nodo, una barra para acercar y alejar la red, una lupa y un botón para ocultar la red de relaciones; y (3) la posibilidad de mostrar distintas gráficas enlazadas. La selección de un nodo abre un espacio lateral donde se muestran todos los atributos que dan información sobre ese nodo y permite abrir en una ventana nueva toda la que está enlazada con una página exterior. La información sobre los autores se obtiene del Directorio de Expertos en el Tratamiento de la Información (EXIT). Se constituye así en una fuente de información secundaria sobre la revista de innegable utilidad, como lo fueron en su día los índices impresos, con la ventaja de que su actualización puede realizarse de forma casi simultánea a la publicación de nuevos números. Los datos obtenidos servirán para la realización de otros análisis de la evolución de la revista, de las temáticas de interés de la profesión y sus actores.

\section{Palabras clave}

Cartografía; Mapas visuales; Visualización de la información; Visualización interactiva; Índices; Recuperación de información; Búsquedas bibliográficas; Análisis de redes; Profesional de la información; EPI; Directorio EXIT; Revistas científicas; Gephi; Comunicación académica; Fuente abierta. 


\begin{abstract}
The process of creating a cartography, an interactive visualization, from the bibliographic data of the contents of the scientific journal Profesional de la información during its almost 30 years of life is presented. The cartography is available on the EPI journal website: $h$ ttps://profesionaldelainformacion.com/cartografia/EPI_GLOBAL/index.html. Its objective is to serve as a navigable index for the quick localization of information of interest to readers as well as a visual map that highlights the characteristics of the knowledge network that these data form through the interconnection of relationships between its nodes. This device has been created by extracting data from the journal's website using web scraping tools and the free software Gephi for network visualization. A graph viewer allows access and manipulation through the web. Navigation through the bibliographic data network is facilitated by different strategies: (1) the use of different colors for each category of node (author, keyword, number, and article); (2) the viewer's search tools, including a node label finder, a zoom bar, a magnifying glass, and a button to hide the network of relationships; and (3) the possibility of displaying different linked graphs. Selecting a node opens a side-space where all the attributes providing information about that node are displayed and that enables a new window to be opened, displaying all the information linked to an external page. The information on the authors is obtained from the Directory of Experts in the Management of Information (EXIT). This tool thus undeniable provides a useful source of secondary information on the journal, as were printed indexes in their day, with the advantage that it can be updated almost simultaneously with the publication of new issues. The data obtained will be used to carry out other analyses of the evolution of the journal, the topics of interest to the profession, and its stakeholders.
\end{abstract}

\title{
Keywords
}

Cartography; Visual maps; Information visualization; Interactive visualizations; Indexes; Information retrieval; Bibliographic search; Network analysis; Profesional de la información; EPI; Directorio EXIT; Scientific journals; Gephi; Scholarly communication; Open source.

\section{Introducción}

Este año 2021 se produce el cierre de un periplo de 30 años en la revista "El profesional de la información", popularmente conocida entre los profesionales como EPI, una de las veteranas en su campo temático. Su nombre oficial desde 2020 es Profesional de la información https://revista.profesionaldelainformacion.com/index.php/EPI

Sirva este trabajo para celebrarlo y homenajear a aquéllos que han estado trabajando por su continuidad durante todo este tiempo, llevándola desde un humilde boletín de noticias profesionales hasta una revista de reconocimiento internacional. Una cartografía de los contenidos publicados estos años, un mapa por el cual desplazarse para observar, recordar o descubrir sus caminos y paisajes, es su objeto. Esta cartografía se muestra mediante una visualización interactiva que permite conocer a los autores que han participado en ella y las áreas temáticas que se han abordado. Está disponible en: https://profesionaldelainformacion.com/cartografia/EPI_GLOBAL/index.html

Permite buscar y navegar entre los datos, que en su mayoría se encuentran interrelacionados.

Ya cuando la revista cumplió 10 años y sus 100 números, se celebró con un número especial que actuaba como bibliografía e índice temático, cuya autora fue Mari-Carmen Marcos:

http://profesionaldelainformacion.com/documentos/bibliografia_iwe_epi_1992_2001.html

La elaboración de un número índice cada cierto tiempo tenía cierta raigambre entre las revistas científicas cuando se publicaban en papel. Eran una fuente de información secundaria de utilidad para las bibliotecas, que hacían el control de las colecciones, y para los usuarios, que buscaban información. Su uso decayó al irse popularizando las bases de datos. Ese número especial no sólo se publicó como un número aparte (enero 2002) sino que tuvo su versión en cd-rom y sus versiones digitales en formatos html, .doc y .pdf, versiones digitales aún hoy disponibles para su descarga.

No es inusual que las revistas celebren hitos de publicación (Araújo; Shideler, 2011; Cooper, 2016a; Peternelj-Taylor, 2014). El esfuerzo de sacarlas adelante y de crear una comunidad de autores y lectores hace que se desee festejar juntos los logros obtenidos, alentar a la comunidad a seguir adelante y recordar lo que hace que merezca la pena el esfuerzo realizado. Es sin duda un momento de satisfacción, pero también de reflexiones para el futuro.

Esta cartografía integra la versión actualizada de la bibliografía del décimo aniversario de EPI, incrementada con los contenidos de dos décadas más de intercambio de información profesional. Complementa otros análisis de la revista como los de Guallar (2019) y López-Robles et al. (2019a; 2019b). Incorpora también las ventajas que la tecnología de la web proporciona en cuanto a interacción y posibilidades de actualización permanente. Concebida como una red de datos bibliográficos circunscrita a una sola publicación, su visualización permite no solo una navegación informativa para localizar artículos de interés, lo cual ya es de un gran valor como herramienta en sí mismo, sino también detectar algunas características del conjunto, como los autores más prolíficos o las materias más populares. 
Conforme fue cumpliendo años, EPI fue adaptándose a diferentes cambios que fueron moldeándola.

Ya en el año 2000 se publicó en la revista una cronología inicial realizada por Estivill y Gascón (2000) ("Historia de El profesional de la información. Cómo catalogar la revista: propuestas de registros". El profesional de la información, v. 9, n. 9): http://profesionaldelainformacion.com/ contenidos/2000/septiembre/5.pdf

La revista mantiene una sucinta cronología que sigue actualizándose y puede encontrarse en:

http://profesionaldelainformacion. com/presentacion.html

La red elaborada -descrita en este artículo- muestra la estructura de conocimiento de las áreas de investigación de $E P I$ reunida durante toda su historia. En total, se extrajeron 4.865 artículos que cubren el período de 1992 a 2021, sus autores y las palabras clave asignadas por ellos a partir del sitio web de la revista. Artículos, autores y palabras clave fueron analizadas con Gephi, que soporta una exploración visual del conocimiento de los datos de la red bibliográfica. Como resultado es posible presentar el panorama general y las principales corrientes de investigación que se publican en esta revista.

En la bibliografía sobre visualización de la información es recurrente la metáfora de la cartografía para describir la exposición de estructuras de conocimiento y navegación (Skupin, 2004). Es una metáfora que se ajusta bien a la idea de una proyección en dos dimensiones de un espacio (real o figurado) que se despliega en tres. De ahí que se hable de cartografía. La interactividad, no siempre necesaria, se entiende como una característica propia de las tecnologías. Esta metáfora es usada también en otras áreas relacionadas con la organización de la información o en bibliometría. Por ejemplo, el modelo Topic maps (ISO/IEC 13250:2003; Colmenero-Ruiz, 2005; Pepper, 2002) que es un estándar para la representación y el intercambio de conocimientos, está también inspirado en

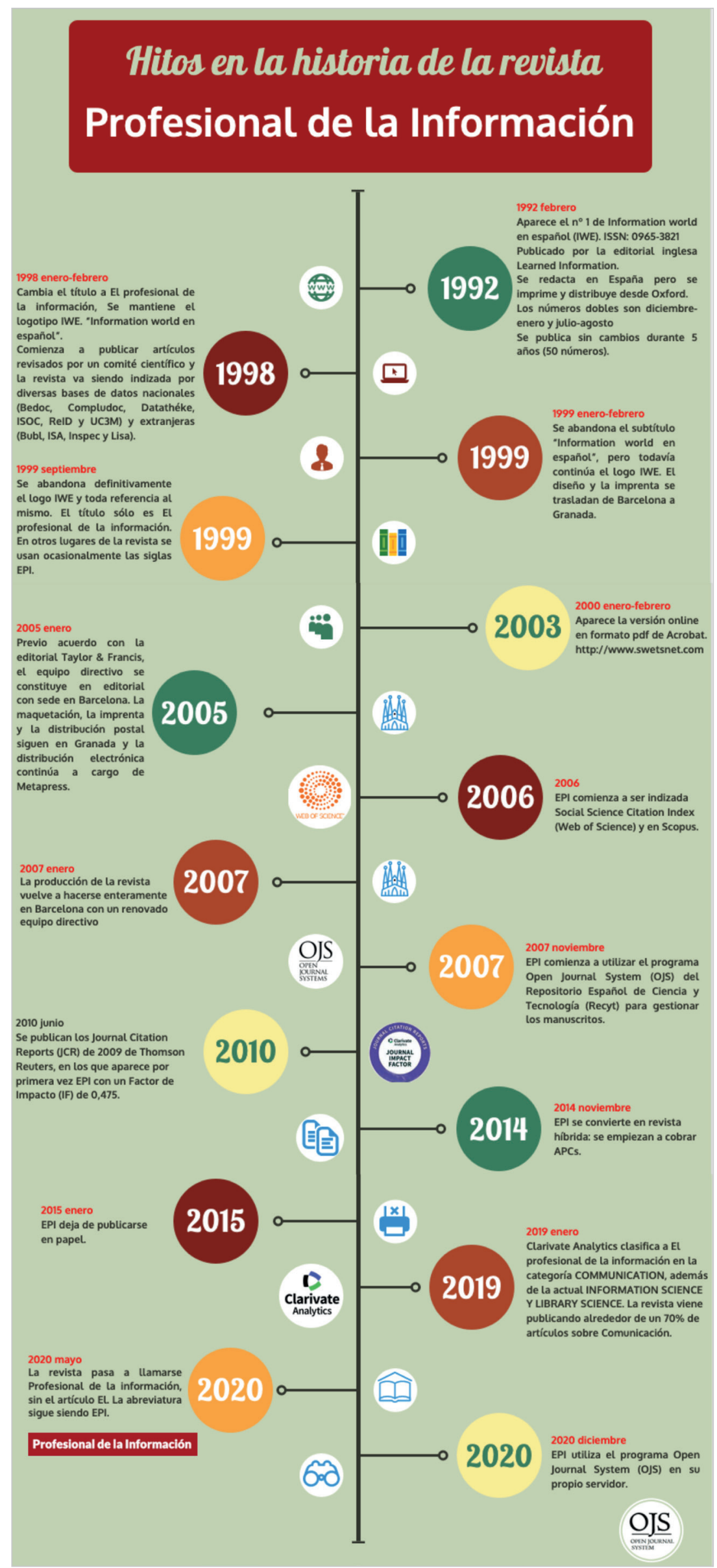

Figura 1. Infografía con los hitos principales de la revista Profesional de la información 
los índices impresos y adoptó el nombre de "mapas de tópicos" porque evocaba la idea de un mapa que ayuda a buscar información, a semejanza de los mapas geográficos que sirven como guías para mostrar la estructura espacial de la superficie terrestre (u otras) y para localizar lugares físicos en ella. La bibliometría la ha adoptado desde que empezó a intentar analizar las estructuras de relaciones y conocimiento que se daban en la ciencia (Chen et al., 2016; Glänzel, 2012). También ha sido usada en otras áreas científicas, especialmente cuando éstas intentan hacer representaciones visuales.

Esto ha dado como resultado una indefinición a la hora de establecer qué es lo que hay detrás de conceptos como visual map, visual mapping, bibliographic mapping, bibliometric mapping, bibliographic cartography, bibliometric cartography, knowledge mapping, etc. (Cooper, 2016b; Parmentier-Cajaiba; Cajaiba-Santana, 2020). Tête (2020) habla de cartographie bibliographique, pero se refiere a cartografía bibliométrica y no a bibliográfica. Por ello es necesario establecer que este trabajo no es de índole bibliométrica, y lo consideremos una cartografía bibliográfica.

Al igual que otros investigadores (Ávila-Toscano et al., 2018; Cheng et al., 2018; Duvvuru; Kamarth; Sivarit, 2012; Gonzales; Noy, 2019; Gouvernet et al., 2020; Lee; Lee, 2019; Nguyen; Park; Yeo, 2021) que utilizan los datos proporcionados por la bibliografía (palabras clave, autores, instituciones...) y los utilizan para analizar la estructura y evolución de las estructuras en red o de conocimiento que forman, hemos utilizado los datos bibliográficos de EPI para mostrar esas relaciones. Sin embargo, no se realiza ningún análisis estadístico de las mismas. El interés radica en la visualización de la estructura en forma de mapas temáticos y las posibilidades informativas y cognitivas que proporciona su exploración.

Esta misma aproximación es la que se realiza en el área del análisis textual con las técnicas de procesamiento del lenguaje natural (PNL) para el reconocimiento de entidades nombradas (named entities recognition), categorías de palabras que representan personas, organizaciones, lugares, fechas..., en un texto. Una vez extraídas pueden analizarse y visualizarse también como una red en forma de grafo (Barros-García, 2015).

\section{Objetivos}

El objetivo principal de este trabajo es la creación y puesta a disposición de una visualización interactiva de la red de conceptos y relaciones formada por los artículos, sus autores y las palabras clave de dichos artículos recogidos en la revista académica Profesional de la información durante sus 30 años de existencia.

De este objetivo principal derivan otros objetivos que no podemos denominar secundarios sino más bien objetivos de continuidad de la investigación:

- que mediante su actualización periódica sirva como sistema permanente de fuente de información para los lectores de la revista;

- la extracción de los datos bibliográficos de todos los números constituye un primer paso que proporciona la materia prima para lograr la realización de estudios adicionales de interés. Uno de ellos es la realización de la cartografía bibliográfica de toda la revista. No existe un conjunto de datos completo que permita hacer estos estudios de forma asequible no experta. Abordarlo requeriría una reorganización de los datos que excede al objetivo de este trabajo;

- los datos permiten también acometer estudios de subconjuntos de materias que han ido evolucionando en la profesión a lo largo del tiempo;

- igualmente, el estudio se puede realizar con los autores y sus dinámicas de colaboración;

- la fusión de este conjunto con otros parecidos de la misma área de conocimiento y temporalidad proporcionaría un conjunto de datos sobre la profesión en España de gran interés, histórico y de tendencias.

\section{Metodología}

Se ha empleado el análisis de redes sociales (ARS, SNA en inglés) (Ávila-Toscano, 2018) como metodología de investigación de las estructuras bibliográficas. El ARS usa las redes y la teoría de los grafos (Andrienko et al., 2020; Otte; Rousseau, 2002) y está en la base de funcionamiento del software empleado para crear la visualización. Aunque su potencial para el análisis estadístico y bibliométrico es evidente, en nuestro caso solo se ha utilizado su funcionalidad para la visualización de la red. El algoritmo empleado ha sido Atlas Force 2 y solo se ha hecho uso de la gradación en cuanto al tamaño de los nodos, proporcional a la cantidad de veces que aparece un determinado concepto.

La cartografía está basada en cuatro categorías de datos de la revista:

1. Los números publicados: 217 números hasta el momento.

2. El total de artículos incluidos en los 217 números: 4.865 artículos.

3. Las palabras clave asignadas por los autores de los artículos: 13.355 palabras clave.

4. Los autores de los artículos y noticias (si se conocen): 2.300 nombres de autor. 

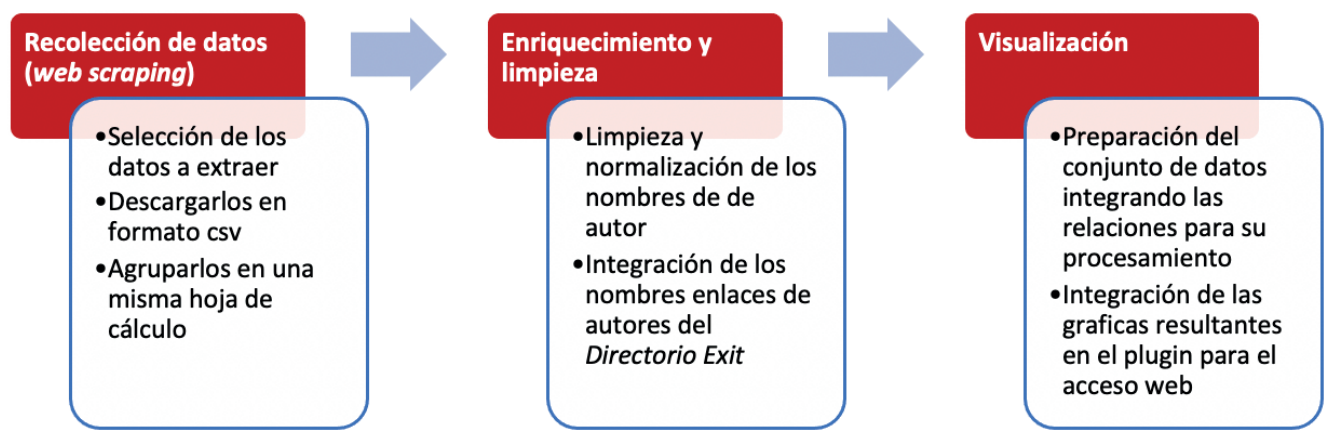

Figura 2. Diagrama de flujo del desarrollo del trabajo

La recolección de estos datos y su procesamiento para ser utilizados posteriormente en el software de visualización ha seguido las fases mostradas en la figura 2.

\subsection{Extracción de los datos}

El primer paso para realizar esta visualización fue obtener los datos bibliográficos de todos los artículos publicados en la revista desde sus inicios. Por desgracia ninguna base de datos que actualmente esté activa dispone de ellos desde el inicio por lo que se han tenido que extraer los datos, depurarlos y posteriormente normalizar los nombres de los autores. La extracción se abordó mediante web scraping (literalmente "raspado web"), técnica que permite extraer la información incluida entre las etiquetas html de una o varias páginas web de forma automática. En nuestro caso las páginas de EPI pueden ser clasificadas en dos grupos:

1) Las que recogen la información más antigua de la revista. Se accede a ellas a partir del índice general disponible en: http://profesionaldelainformacion.com/contenidos.html

A partir de una página índice o sumario de cada número se accede a la noticia o artículo bien a través de un enlace a otra página html o a un archivo pdf en años más recientes. Aunque están todos los artículos en abierto hasta tres años antes del último número publicado, la realidad es que los pdfs han sido la elección para todo lo publicado desde el no 6 del vol. 16 de 2007 por la facilidad de obtención de parte de los datos de interés.

2) Las páginas de los archivos de la revista soportada por el programa OJS:

https://revista.profesionaldelainformacion.com

En ella se ofrece un índice general para el número que da acceso a páginas individuales para cada artículo. Cada página individual ofrece datos bibliométricos en mayor número, de interés para nuestro trabajo. Ha sido la fuente de elección para la información disponible desde finales de 2007. Tiene además la ventaja de aportar los datos necesarios de los números con embargo sin necesidad de ser suscriptor y de una forma más sencilla que en el caso anterior.

Aunque ambos grupos tienen un formato html su estructura y contenidos son distintos por lo que hubo que abordar la extracción de forma diferente en una y otra. El segundo grupo, más actual, aporta datos no disponibles en los primeros años, como las palabras clave o la forma de citar el artículo. Las palabras clave de muchos de los artículos de la primera época tuvieron que ser recogidas a mano directamente de los pdfs puesto que no aparecían en la página web del índice.

Los datos de ambos grupos se extrajeron con extensiones del navegador Chrome que permiten realizar web scraping sin necesidad de conocimientos técnicos, tras realizar pruebas con varios disponibles en el Chrome web store. Para las páginas del grupo 1 se eligió Grepsr:

https://chrome.google.com/webstore/detail/grepsr-web-scraping-tool/hjdijkhlfpeafghibmiabeofkiicdnjm?hl=es

Para las del grupo 2 nos decantamos por Web Scraper:

https://chrome.google.com/webstore/detail/web-scraper-free-web-scra/jnhgnonknehpejjnehehllklip/mbmhn

menos sencilla de manejo pero que permite incluir los URLs de todos los artículos y recolectar automáticamente la información escogida en mayor cantidad.

La suma de los resultados obtenidos en forma de archivos en formato csv se reunieron en un archivo único en formato Excel. Este archivo es adecuado para proceder a la limpieza de datos, para ser enriquecido con datos procedentes de otras fuentes y para ser procesado por el software de visualización elegido por el equipo. La limpieza más básica se realiza con el mismo Excel: buscar y reemplazar caracteres no deseados como corchetes o paréntesis en los campos de nombre de autor, separar autores y orcids, etc.

La bibliografía realizada por Mari-Carmen Marcos de los 10 primeros años aportó la autoría de muchas noticias de los primeros años que no aparecían en la web.

El primer paso para realizar esta visualización fue obtener los datos bibliográficos de todos los artículos publicados en la revista desde 1992, a partir de distintas fuentes 


\subsection{Enriquecimiento y normalización}

Un problema que emerge al tener recogidos todos los nombres de los autores tal y como fueron registrados en su día en los artículos es su falta de normalización. Aunque en algunos casos es fácil detectar las formas alternativas, no siempre es así. El número orcid es precisamente un sistema ideado como forma de normalizar los autores, problema largamente conocido en la profesión. La revista lleva incorporando este número para la identificación de los autores desde el volumen 21, número 6 del año 2012, lo que deja a la mayoría de los autores anteriores sin esta identificación. Hay que anotar que $E P I$ fue la primera revista española en incorporar los orcids de los autores.

EPI enlaza a todos los autores con su ficha correspondiente en el Directorio de Expertos en el Tratamiento de la Información (Directorio EXIT) (Orduña-Malea; Rodríguez-Gairín; Baiget, 2007), si ésta existe, desde el volumen 17, número 6 del año 2008:

https://www.directorioexit.info

Esto nos proporcionaba casi 4 años más de autores identificados que con orcid, unos 13 años en total. Esta identificación no la hace en las páginas de la revista de la plataforma OJS (grupo 2 según clasificamos anteriormente) sino en las páginas índice (grupo 1). Como las páginas del grupo 1 no se habían usado para recolectar datos en estos años hubo que realizar una recolección específica para extraer los nombres de los autores y su enlace al Directorio EXIT.

La información del Directorio es una información profesional que no hace hincapié en la faceta estrictamente de autor académico, como es el caso de orcid. La edita el propio autor y su inclusión en el conjunto de datos enriquece la información proporcionada por la visualización del mismo.

Los autores del período 1992-2008 de los que no se disponía del enlace EXIT se han intentado localizar de forma manual. Téngase en cuenta que muchos de ellos ya estaban identificados porque volvieron a escribir en la revista. Otros tantos no y fueron estos los que se buscaron. Dado que los nombres de los autores no están normalizados, ni dentro de la revista, ni con el Directorio EXIT, todo ese trabajo y la interconexión con los que no tenían el enlace hecho ha requerido un esfuerzo importante de trabajo no automatizado apoyado por la herramienta OpenRefine.

https://openrefine.org

Se ha mantenido, para cada autor, una sola forma del nombre procedente de los artículos de la revista y ésta de preferencia a la del directorio. Este proceso es el que más errores puede haber acumulado, dado su fuerte componente manual.

Se ha seguido el criterio aplicado por la revista en los últimos años de escribir los nombres de autor en un formato internacional (nombres y apellidos unidos con un guion), recuperando el segundo apellido cuando ha sido posible, incluso cuando no se publicaron así. En la decisión ha pesado la consideración "histórica" de esta cartografía, pues muchos autores con un solo apellido pasados unos años resultan ilocalizables.

Los editores de EPI han aportado casi un millar de fotos de autores que no tenían ficha EXIT, tanto a partir del stock que poseen como mediante búsquedas por la Web.

Tabla 1. Datos bibliográficos de EPI extraídos para ser analizados

\begin{tabular}{|c|c|}
\hline Identificador del número & $\begin{array}{l}\text { Identificador único del número de la revista. Incluye el no de volumen, el número del número y el año de } \\
\text { publicación. Ej.: v. } 13, \text { n. } 5 \text { (2004). } \\
\text { Nota: Aunque los primeros años no tienen número de volumen se le ha incorporado para dar uniformidad y } \\
\text { facilitar la ordenación en la hoja de cálculo }\end{array}$ \\
\hline Identificador del artículo & $\begin{array}{l}\text { Identificador único del número: Se compone del identificador del número de la revista condensado más un } \\
\text { número de artículo, que se le ha asignado correlativamente desde el primero publicado. Ej: v. 13, n. } 5 \text { (2004) } \\
\text { - Art. } 3090\end{array}$ \\
\hline Año de publicación & Se publica un volumen por año \\
\hline Enlace al número (índice) & URL del número \\
\hline Enlace a la imagen de portada & URL de la imagen de la portada de la revista \\
\hline Título artículo & Título del artículo \\
\hline Enlace artículo & URL del artículo \\
\hline Autores & Autores del artículo. Separados tras la recolección en columnas individuales \\
\hline Información EXIT & URL de la ficha del autor en el Directorio EXIT \\
\hline DOI & URL del DOI. Se aplicó desde enero de 2007 (v. 16, n. 1) \\
\hline Palabras clave & Recogidas en conjunto y separadas posteriormente en columnas \\
\hline Resumen & Resumen del artículo, si existe \\
\hline Orcid & Identificador orcid de los autores. Este dato lleva recolectándose desde noviembre de 2012 (v. 21, n. 6) \\
\hline Cita & Recoge la citación recomendaba el artículo tal como aparece en la ficha del artículo en OJS \\
\hline Sección & Sección en la que se enmarca del artículo considerado \\
\hline Relacionados & Artículos relacionados destacados en la ficha del artículo en OJS \\
\hline Enlace al pdf & URL del artículo en formato pdf si se encuentra disponible \\
\hline
\end{tabular}


Para aplicar la perspectiva de género al conjunto de datos se incorporó el dato del sexo, asignado a partir del nombre y fotografía (si estaba disponible). La distribución resultante fue 1.217 autoras y 1.310 autores varones. Dado que esta ha sido una profesión tradicionalmente muy feminizada esta distribución cercana al 50\% para ambos sexos, aunque inclinada hacia el masculino ( $48 \%$ y $52 \%$ ), resultaba un tanto chocante. La realidad es que está mostrando lo que ocurre principalmente en el ámbito académico más que en el puramente profesional.

Los datos tabulados extraídos se muestran en la tabla 1.

\subsection{Visualización}

Una vez obtenida la tabla de datos tras el proceso de recogida, limpieza y emparejamiento se procedió a prepararlos para la visualización propiamente dicha.

El software utilizado ha sido Gephi (Bastian; Heymann; Jacomy, 2009): https://gephi.org

Gephi es un programa para visualizar, explorar y comprender todo tipo de gráficos y redes (Cherven, 2015), que sirve como complemento a los estudios estadísticos tradicionales. Pero, además de facilitar los estudios métricos, ayuda a mostrar las estructuras subyacentes de relaciones entre objetos, o sea estructuras de conocimiento con su presentación cartografiada. Esta posibilidad permite aprovechar las ventajas que la representación visual del conocimiento tiene, especialmente en grandes conjuntos de datos.

Es gratuito y de código abierto. Gephi normalmente es una aplicación para la manipulación de los gráficos por un solo usuario en su instalación local.

Pero existe un visor que permite exportar a la web las gráficas realizadas con Gephi, realizado por Velt (2011). Se llama gexf.js y está disponible en Github https://github.com/raphv/gexf-js

Instalado en un servidor es el que permite la interactividad para múltiples usuarios.

Este visor admite la inclusión de varias gráficas, una facilidad que puede usarse de varias formas. En este trabajo hemos elegido usarla para proporcionar, además de la gráfica global que incluye todas las categorías de nodos (autores, palabras clave, números y artículos), otras gráficas que actúan a modo de filtro de la información, en las que solo se muestran las relaciones importantes de una de las categorías.

Para ser manejados con Gephi los datos deben disponerse de un modo preciso: identificando cada categoría de nodos y sus atributos y creando las relaciones existentes entre ellos. Para ello pueden usarse varios programas, entre los que se encuentra Excel.

Esta parte de preparación de datos para su manipulación por el programa también implica un componente de gestión manual. Cada gráfica a ser visualizada, además, requiere su conjunto de tablas específico.

\section{Resultados}

La visualización interactiva construida con Gephi y el visor gexf.js es navegable por un número muy elevado de nodos (más de 20.000), unidos por sus relaciones. Para facilitar el movimiento por esta extensa red de datos bibliográficos el visor incorpora distintas formas de ayuda al usuario.

Una de ellas es el uso de elementos visuales de comunicación. Cada categoría de nodo (autor, palabra clave, número de revista y artículo) se diferencia por el color. Se ha evitado el uso del rojo y el verde juntos para evitar que afecte a la forma de daltonismo más frecuente. A su vez su tamaño está determinado, en general, por el número de sus ocurrencias. Las excepciones se realizan por criterios de facilitación cognitiva o por razones de sesgo conocido. Estas posibilidades distan mucho de ser solo estéticas, sirven como recurso informativo que ayudan a diferenciarlos de forma rápida y a resaltar su peso en la red (figura 3).
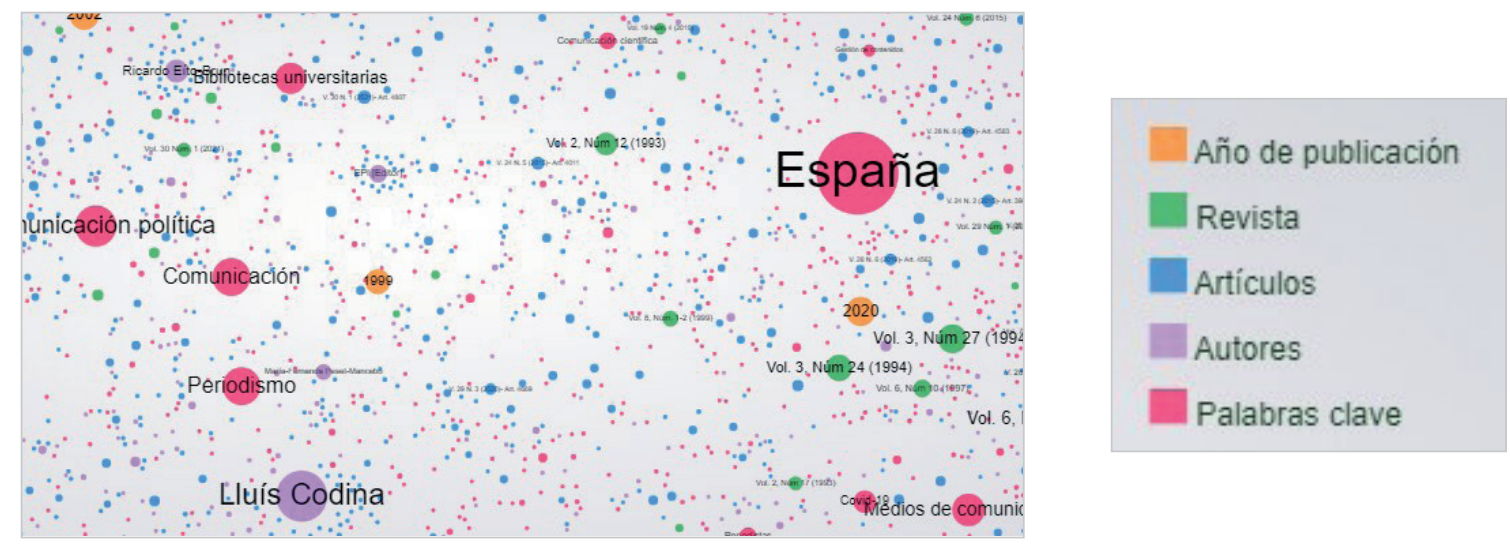

Figura 3. Códigos visuales de color y tamaño usados en la visualización 

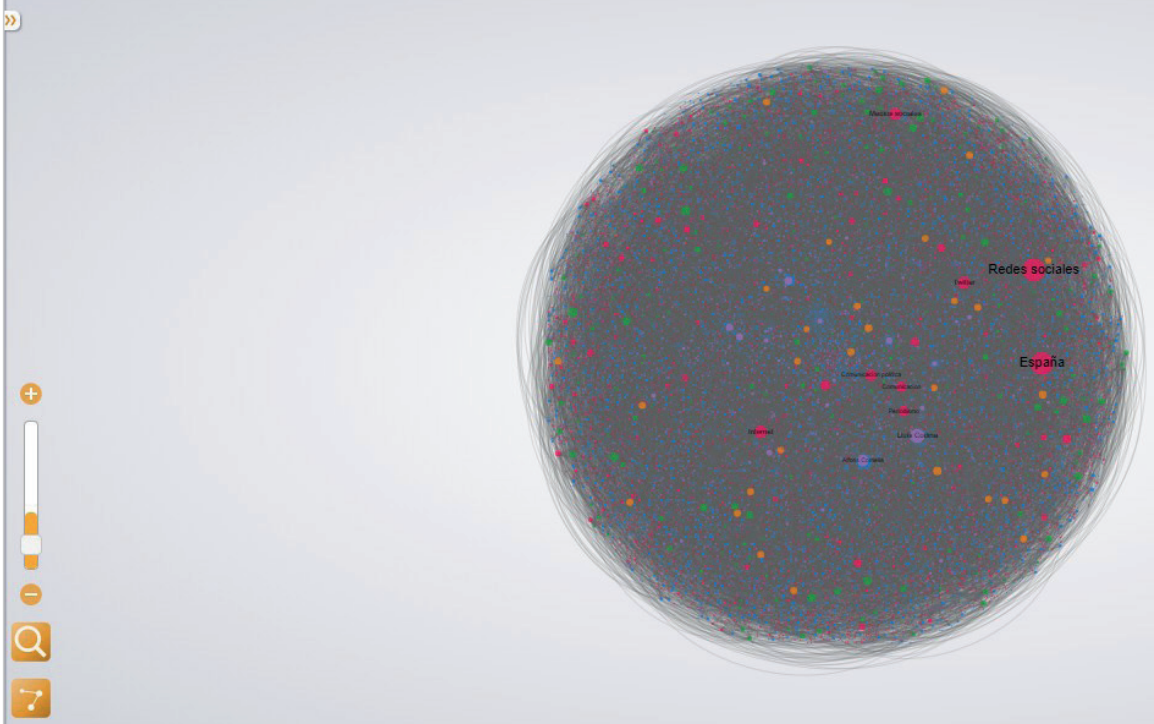

Cartografia GLOBAL

Año de publicación

Revista

Aarticulos

Autores

Palabras clave

$\oplus$

Figura 4. Interfaz web de la red de datos bibliográficos de Profesional de la información

Como los nodos tipo "artículo" solo tienen una ocurrencia, el gráfico muestra 4.865 puntos de color en el fondo formando una nube densa que se suma al resto de nodos y a las líneas de enlace que representan los vínculos entre los distintos nodos (figura 4). Es posible ocultar y mostrar esta nube a voluntad.

Para poder navegar por la red el visor incorpora un segundo tipo de ayuda: la caja de búsqueda, de gran utilidad si ya se tiene una idea de lo que se busca. Usando texto libre ofrece resultados ordenados de nombre de autor, palabras clave, números y artículos de la revista, pudiendo seleccionar lo más cercano o adecuado a nuestro interés (figura 5).

Incorpora también una barra para realizar zoom, que acerca y aleja la red de nodos. El ratón posibilita el movimiento en los ejes $x$ e y. Si se necesita más detalle en alguna zona puede pulsarse en el icono lupa transformándose así el cur-

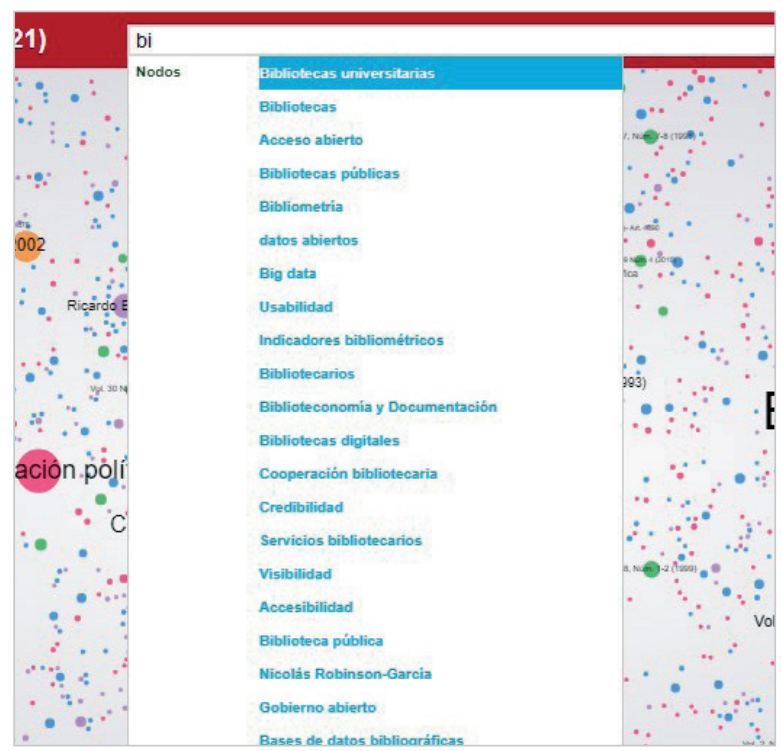

Figura 5. Resultados predictivos de la caja de búsqueda al introducir "bi"

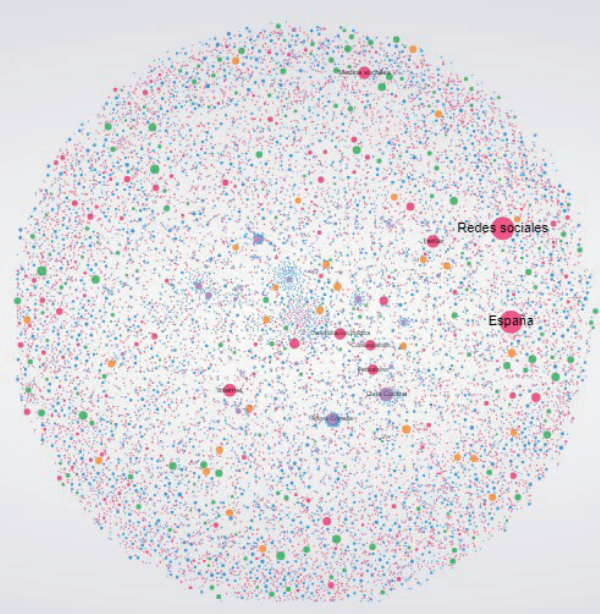

Cartografia GLOBAL

Waño de publicación

Revista

Articulos

Autores

Palabras clave

Figura 6. Interfaz web de la red de datos bibliográficos de Profesional de la información sin el enjambre de enlaces 
sor en una gran lupa para explorar rápidamente un área de la pantalla. Si se desea también es posible ocultar la red de relaciones como ya se indicó (figura 6).

Al pulsar en alguno de los nodos de la nube se puede acceder a la información disponible sobre él, y consultar el artículo completo en el sitio web de la revista. Esta información se muestra en la parte izquierda de la página, en un marco que puede abrirse o cerrarse a voluntad (figura 7).

Por último, es posible mostrar en el mismo sitio distintas gráficas enlazadas entre sí. En nuestro caso hemos elegido gráficas que permiten mostrar la red filtrada por autor, materia y año de publicación (que coincide con el volumen). Desde

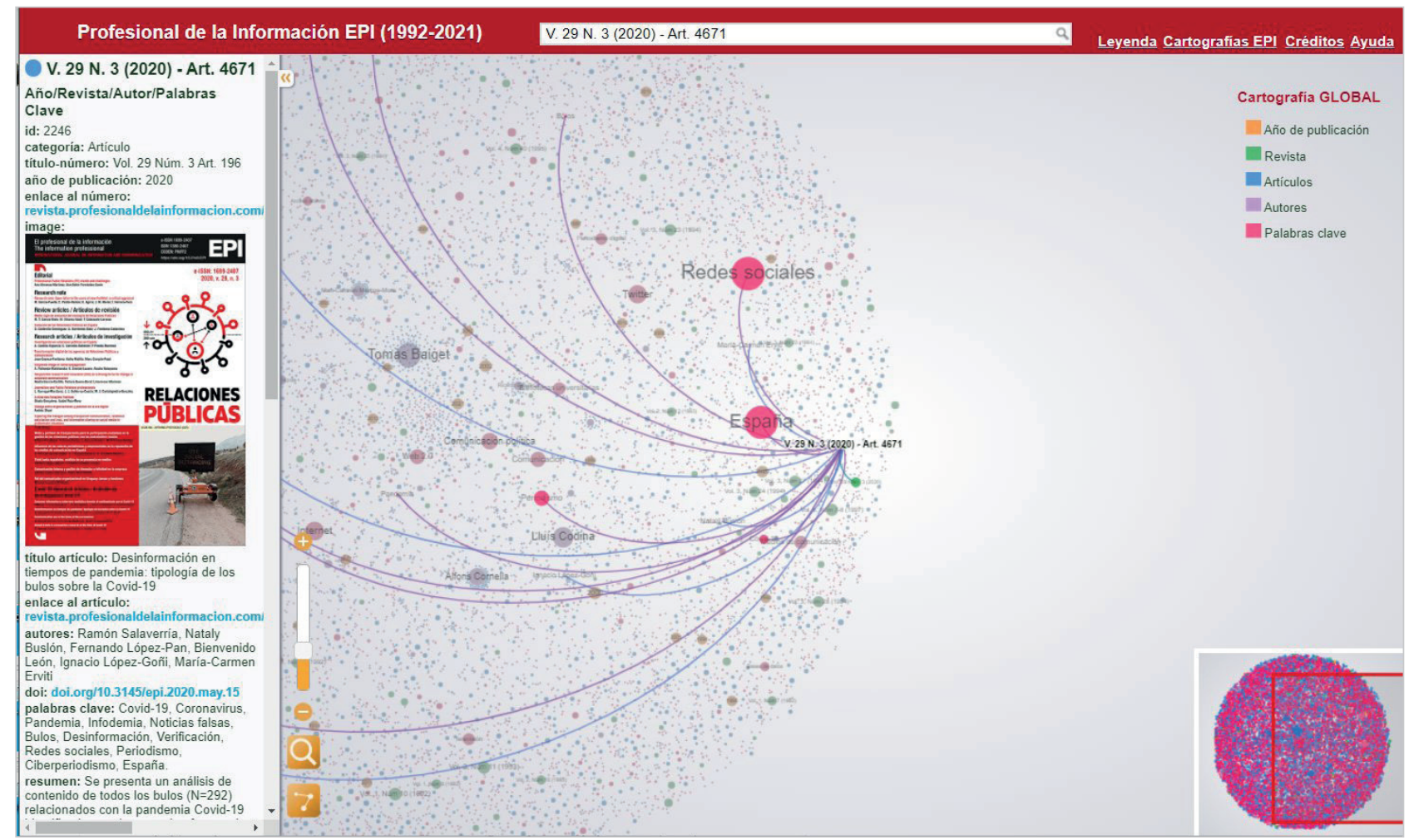

Figura 7. Marco lateral abierto mostrando la información disponible sobre el nodo seleccionado, que en este ejemplo corresponde a un artículo.

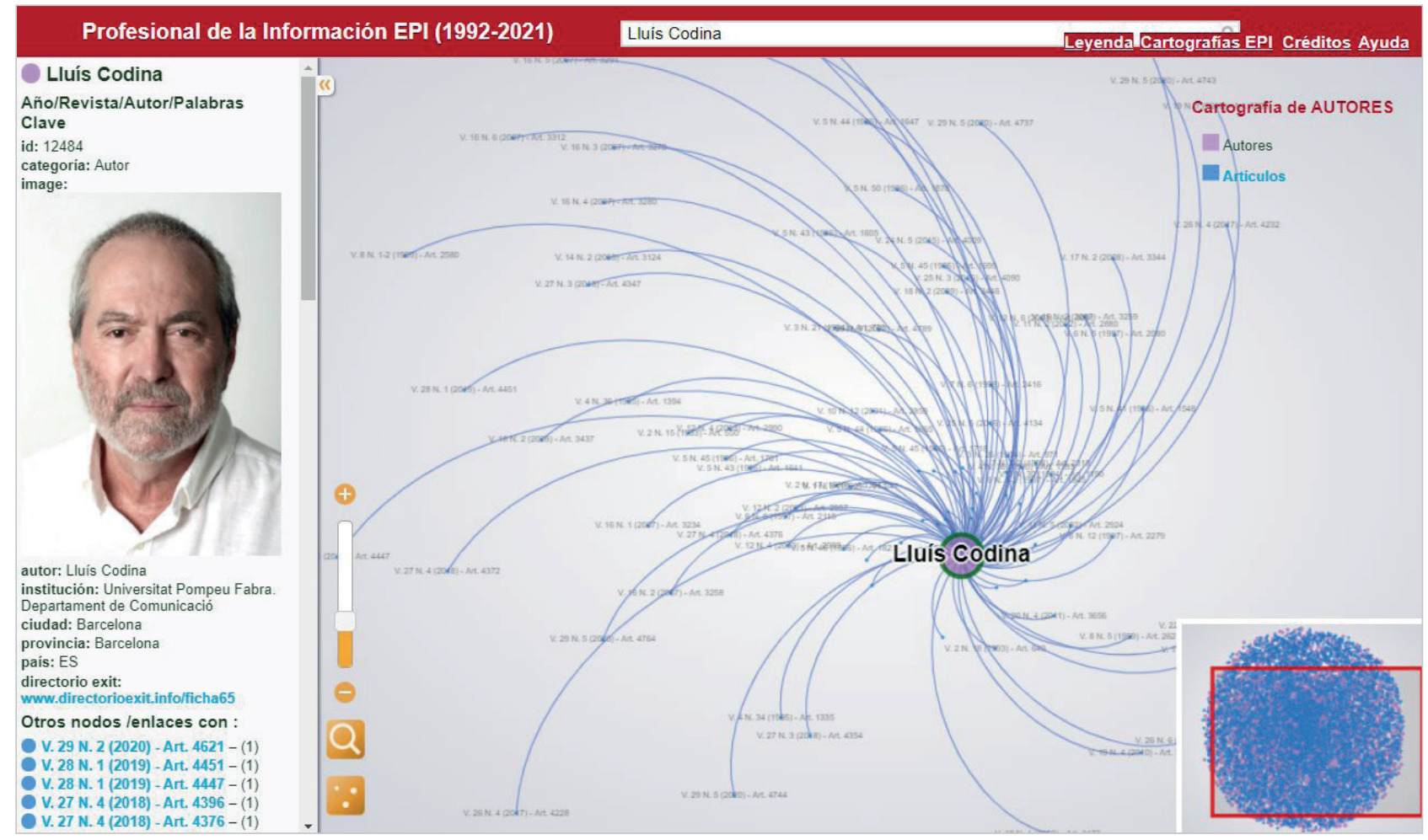

Figura 8. Visualización de la red de autores 
el enlace Cartografías EPI que aparece en la parte superior derecha de la página se enlaza con otras páginas que muestran en exclusiva la red de autores, palabras clave o año/volumen de la revista. Con ello el número de nodos se reduce y se pone de relieve únicamente la información relativa a cada categoría. Como resultado sus características se ven resaltadas (figuras 8,9 y 10 ).
La visualización interactiva construida con Gephi y el visor gexf.js es navegable por un número muy elevado de nodos (más de 20.000), unidos por sus relaciones

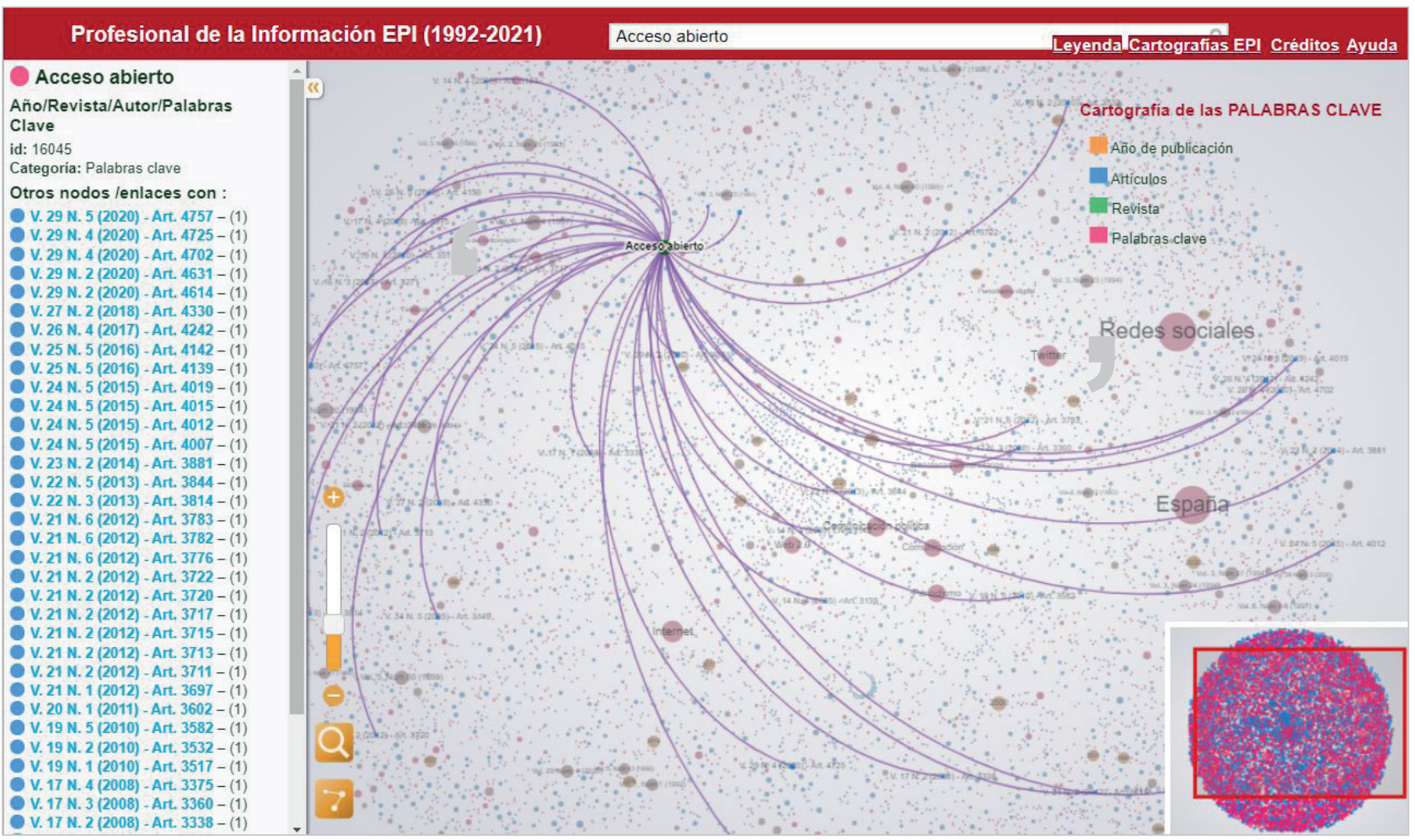

Figura 9. Visualización de la red de palabras clave

\section{Profesional de la Información EPI (1992-2021) Vol. 29 Núm. 4 (2020)}

Leyenda Cartografias EPI Créditos Ayuda

Vol. 29 Núm. 4 (2020) Año/Revista/Autor/Palabras Clave

id: 10005

Categoria: Vol. Num. Revista

Otros nodos /enlaces con :

2020 - (1)

V. 29 N. 4 (2020) - Art. 4734 - (1)

v. 29 N. 4 (2020) - Art. 4733 - (1)

V. 29 N. $4(2020)$ - Art. 4731 - (1)

V. 29 N. $4(2020)$ - Art. $4730-(1)$

V. 29 N. 4 (2020)-Art. 4729 - (1)

V. 29 N. $4(2020)$ - Art. $4728-(1)$

V. 29 N. $4(2020)$ - Art. $4728-(1)$
V. 29 N. $4(2020)$ - Art. $4727-(1)$

V. 29 N. 4 (2020) - Art. $4726-(1)$

V. 29 N. $4(2020)$ - Art. $4725-(1)$

V. 29 N. 4 (2020) - Art. 4724 - (1)

V. 29 N. 4 (2020)-Art. 4723 - (1)

V. 29 N. 4 (2020) - Art. $4722-(1)$

V. 29 N. 4 (2020) - Art. 4721 - (1)

v. 29 N. 4 (2020) - Art. 4720 - (1)

V. 29 N. 4 (2020) - Art. 4719 - (1)

V. 29 N. 4 (2020) - Art. 4718 - (1)

V. 29 N. 4 (2020) - Art. 4717 - (1)

V. 29 N. 4 (2020) - Art. 4716 - (1)

V. 29 N. 4 (2020) - Art. 4715 - (1)

V. 29 N. 4 (2020) - Art. 4714 - (1)

V. 29 N. 4 (2020) - Art. 4713 - (1)

V. 29 N. 4 (2020) - Art. 4712 - (1)

V. 29 N. 4 (2020) - Art. 4711 - (1)

V. 29 N. 4 (2020) - Art. 4710 - (1)

V. 29 N. 4 (2020) - Art. 4709 - (1)

V. 29 N. 4 (2020) - Art. 4708 - (1)

V. 29 N. 4 (2020) - Art. 4707 - (1)

V. 29 N. 4 (2020) - Art. 4706 - (1)

V. 29 N. 4 (2020) - Art. 4705 - (1)

v. 29 N. 4 (2020) - Art. 4704 - (1)

V. 29 N. 4 (2020) - Art. 4703 - (1)

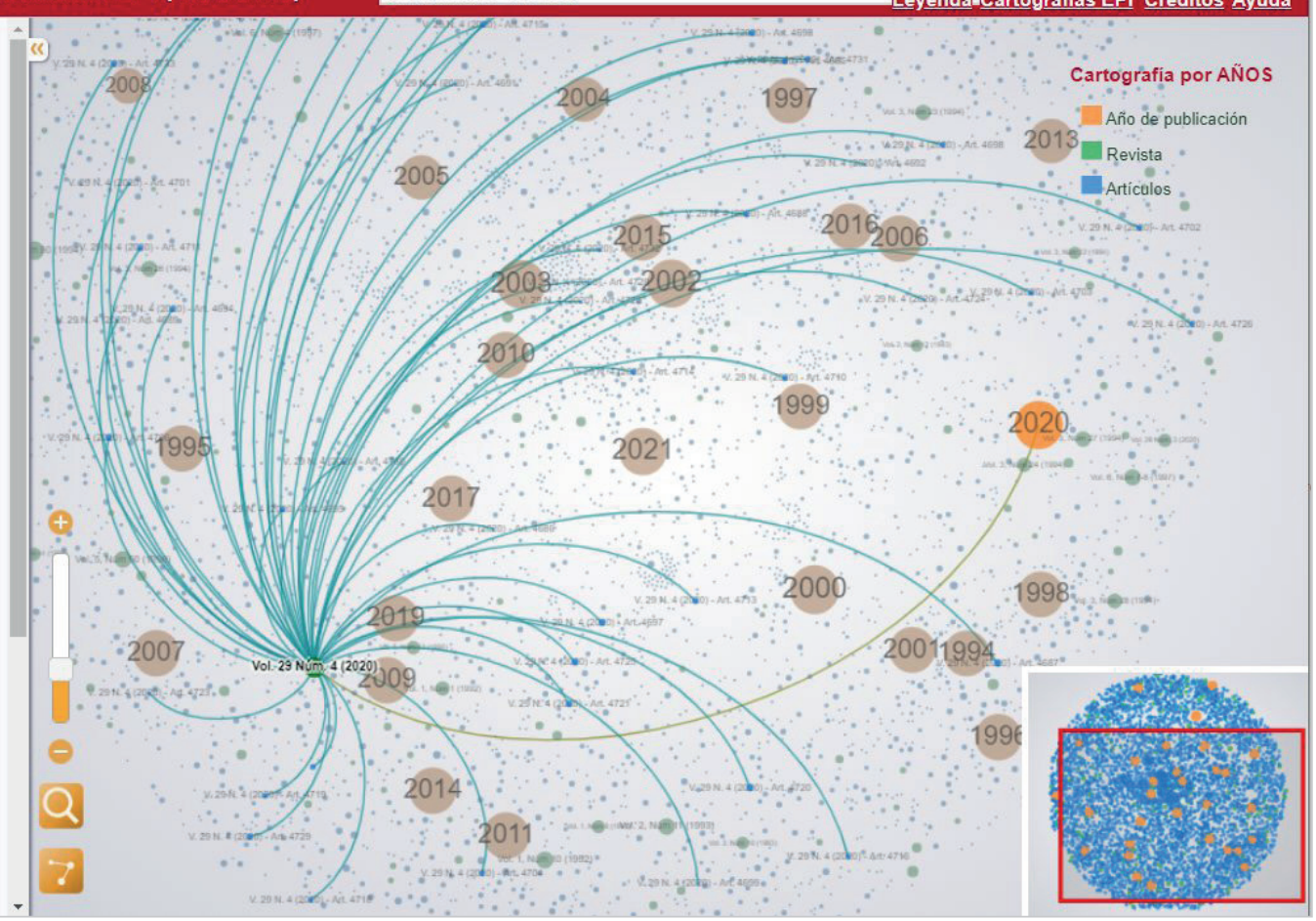

Figura 10. Visualización de la red de volúmenes/artículos de la revista 
Los elementos de navegación descritos se muestran en la tabla 2.

Hay disponible una página de ayuda que explica las principales características de la interfaz y su manejo para facilitar la navegación.

\section{Conclusiones}

Este trabajo de investigación aplicada muestra dos dimensiones de interés. Una es la aquí presentada, una visualización interactiva que hace la función de índice de todo lo publicado por la revista, una fuente de información renovada. Este índice permite localizar los artículos publicados por la revista por temática o por autor, conocer datos sobre los autores y detectar algunas características de su estructura de conocimiento gracias a la proporcionalidad de los nodos y las relaciones entre ellos. Es susceptible de mejora en cuanto a la gestión de los orcids, elección de los nombres normalizados y otras informaciones que pudieran interesar a los usuarios. Otra posibilidad sería crear un vocabulario controlado a partir de las palabras clave.
Tabla 2. Elementos de navegación del software de visualización utilizado

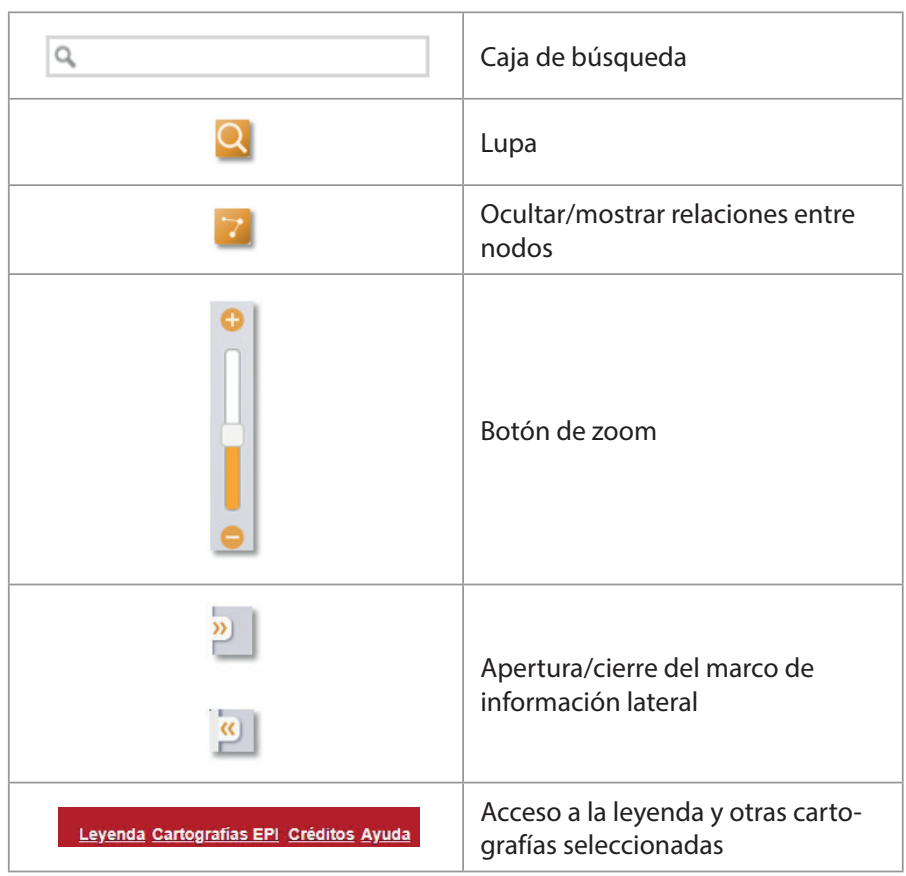

La otra dimensión emana de la posibilidad de usar el conjunto de datos extraído y limpio como base para abordar otros estudios complementarios.

Creemos que las visualizaciones de mapas de conocimiento pueden ser una baza importante en la búsqueda de información, especialmente en conjuntos específicos y relativamente limitados de documentos, favoreciendo a su vez la comprensión de la estructura del propio conjunto.

La utilidad del índice para la navegación y localización de información publicada por la revista es innegable, tanto para autores como para las palabras claves de interés, reuniendo y dando acceso rápido a los artículos que el usuario desea consultar.

\section{Nota}

Los autores agradecerán les sea notificado cualquier error u omisión detectado por los usuarios de la Cartografía EPI.

\section{Bibliografía}

Andrienko, Natalia; Andrienko, Gennady; Fuchs, Georg; Slingsby, Aidan; Turkay, Cagatay; Wrobel, Stefan (2020). "Visual analytics for understanding relationships between entities". In: Visual analytics for data scientists. Cham: Springer, pp. 201-218.

https://doi.org/10.1007/978-3-030-56146-8_7

Araújo, Rafael J.; Shideler, Geoffrey (2011). "Celebrating 60 years of publication of the Bulletin of marine science: A bibliometric history (1951-2010)". Bulletin of marine science, v. 87, n. 4, pp. 707-726.

https://doi.org/10.5343/bms.2011.1090

Ávila-Toscano, José-Hernando; Romero-Pérez, Ivón-Catherine; Marenco-Escuderos, Ailed; Saavedra-Guajardo, Eugenio (2018). "Identification of research thematic approaches based on keywords network analysis in Colombian social sciences". In: Thomas, Ciza (ed.). Data mining. London: InTechOpen. ISBN: 9781789235975 https://doi.org/10.5772/intechopen.76834

Barros-García, Benamí (2015). "Las aportaciones de la visualización del texto al estudio del texto literario". Tonos digital. Revista electrónica de estudios filológicos, v. 1, n. 28. http://www.tonosdigital.com/ojs/index.php/tonos/article/view/1210/738

Bastian, Mathieu; Heymann, Sebastien; Jacomy, Mathieu (2009). "Gephi: an open source software for exploring and manipulating networks". In: Third international AAAl conference on weblogs and social media, pp. 361-362. https://ojs.aaai.org/index.php/ICWSM/article/view/13937

Chen, Xiuwen; Chen, Jianming; Wu, Dengsheng; Xie, Yongjia; Li, Jing (2016). “Mapping the research trends by co-word analysis based on keywords from funded project". Procedia computer science, v. 9, pp. 547-555.

https://doi.org/10.1016/j.procs.2016.07.140 
Cherven, Ken (2015). Mastering Gephi network visualization: Produce advanced network graphs in Gephi and gain valuable insights into your network datasets. Birmingham, UK: Packt Publishing. ISBN: 9781783987344 https://www.packtpub.com/product/mastering-gephi-network-visualization/9781783987344

Colmenero-Ruiz, María-Jesús (2005). "Introducción al modelo Topic Maps (ISO/IEC13250:2003)". RDBCl: Revista digital de biblioteconomia e ciência da informação, v. 3, n. 2, pp. 77-102.

https://doi.org/10.20396/rdbci.v3i1.2055

Cooper, I. Diane (2016a). "An anniversary and you can help". Journal of the Medical Library Association, v. 104, n. 1, pp. 1-2. https://doi.org/10.3163/1536-5050.104.1.001

Cooper, I. Diane (2016b). "What is a 'mapping study'?”. Journal of the Medical Library Association, v. 104, n. 1, pp. 76-78. https://doi.org/10.3163/1536-5050.104.1.013

Duvvuru, Arjun; Kamarthi, Sagar; Sultornsanee, Sivarit (2012). “Undercovering research trends: Network analysis of keywords in scholarly articles". In: Ninth International conference on computer science and software engineering (JCSSE), pp. 265-270.

https://doi.org/10.1109/JCSSE.2012.6261963

Estivill, Assumpció; Gascón, Jesús (2000). "Historia de El profesional de la información. Cómo catalogar la revista: propuestas de registros". El profesional de la información, v. 9, n. 9.

http://profesionaldelainformacion.com/contenidos/2000/septiembre/5.pdf

Glänzel, Wolfgang (2012). "Bibliometric methods for detecting and analysing emerging research topics". El profesional de la información, v. 21, n. 1, pp. 194-201.

https://doi.org/10.3145/epi.2012.mar.11

Gonzales, Audilio; Noy, Claire (2019). "Vers de nouvelles formes de collaboration et de recherche d'informations: du référencement de mots-clés à la visualisation des connaissances". In: Roxin, Ioan; Saleh, Imad; Bouhaï, Nasreddine; Leleu-Merviel, Sylvie; Jeanneret, Yves; Zacklad, Manuel; Massou, Luc. (coords.). H2PTM'19: De I'hypertexte aux humanités numériques, pp. 177-199. London, UK: ISTE Group. ISBN: 9781784066512

https://www.istegroup.com/fr/produit/h2ptm19

Gouvernet, Brice; Hentati, Yassamine; Rebelo, Maria-Teresa; Rezrazi, Amine; Sebbe, Fabrice; Combaluzier, Serge (2020). "Porn studies or pornology? Network analysis of the keywords of science". Porn studies, v. 7, n. 2, pp. $228-246$. https://doi.org/10.1080/23268743.2019.1615378

Guallar, Javier (2019). "La revista El profesional de la información (EPI). Algunas claves". En: IX Congreso universitario de comunicación y tecnología (Comunica2), UPV, Gandia (España), 14-15 noviembre.

http://eprints.rclis.org/39679

ISO/IEC 13250:2003. Information technology - SGML applications - Topic maps.

Lee, MiYoung; Lee, Jungmin (2019). "Research trends in Journal of fashion business - A social network analysis of keywords in fashion marketing and design area-". Journal of fashion business, v. 23, n. 3, pp. 51-66.

https://doi.org/10.12940/JFB.2019.23.3.51

Leydesdorff, Loet (2016). "Advances in science visualization: social networks, semantic maps, and discursive knowledge". In: Cronin, Blaise; Sugimoto, Cassidy (eds.). Bibliometrics and beyond: Metrics-based evaluation of scholarly research (pp. 1-25). Cambridge: The MIT Press.

https://arxiv.org/abs/1206.3746

López-Robles, José-Ricardo; Guallar, Javier; Gamboa-Rosales, Nadia-Karina; Otegi-Olaso, José-Ramón; Cobo, Manuel-Jesús (2019a). "Mapa de la estructura intelectual de El profesional de la información de 2014 a 2018 ". Hipertext. net, n. 19, pp. 115-125.

https://raco.cat/index.php/Hipertext/article/view/360359

López-Robles, José-Ricardo; Guallar, Javier; Otegi-Olaso, José-Ramón; Gamboa-Rosales, Nadia-Karina (2019b). “El profesional de la información (EPI): bibliometric and thematic analysis (2006-2017)”. El profesional de la información, v. 28, n. 4, e280417.

https://doi.org/10.3145/epi.2019.jul.17

Nguyen, Thi-Le-Hang; Park, Sung-Hoon; Yeo, Gi-Tae (2021). "Keyword network analysis: Uncovering research trends on the Northern Sea route". The Asian journal of shipping and logistics, v. 37, n. 3, pp. 231-238.

https://doi.org/10.1016/j.ajsl.2021.06.001

Orduña-Malea, Enrique; Rodríguez-Gairín, Josep-Manuel; Baiget, Tomàs (2007). "Directorio de expertos en el tratamiento de la información (EXIT). Análisis de uso". El profesional de la información, v. 16, n. 5, pp. 497-509.

https://doi.org/10.3145/epi.2007.sep.13 
Otte, Evelien; Rousseau, Ronald (2002). Social network analysis: a powerful strategy, also for the information sciences. Journal of information science, v. 28, n. 6, pp. 441-453.

https://doi.org/10.1177/016555150202800601

Parmentier-Cajaiba, Aura; Cajaiba-Santana, Giovany (2020). "Visual maps for process research: Displaying the invisible". M@n@gement, v. 23, n. 4,pp.65-79.

https://doi.org/10.37725/mgmt.v23i4.4501

Pepper, Steve (2002). The TAO of topic maps: finding the way in the age of infoglut. Revision of the original from 2000. http://www.ontopia.net/topicmaps/materials/tao.html

Peternelj-Taylor, Cindy (2014). "Celebrating the $10^{\text {th }}$ anniversary of the Journal of forensic nursing". Journal of forensic nursing. January/March, v. 10, n. 1, pp. 1-3.

https://doi.org/10.1097/JFN.0000000000000023

Skupin, André (2004). "The world of geography: Visualizing a knowledge domain with cartographic means". Proceedings of the National Academy of Sciences (PNAS), v. 6, n. 101 (suppl. 1), pp. 5274-5278.

https://doi.org/10.1073/pnas.0307654100

Tête, Caroline (2020). "La littérature scientifique française en soins palliatifs: Cartographie bibliographique". Médecine palliative, v. 19, n. 1, pp. 36-40.

https://doi.org/10.1016/j.medpal.2019.08.010

Velt, Raphaël (2011). gexf-js.

https://github.com/raphv/gexf-js

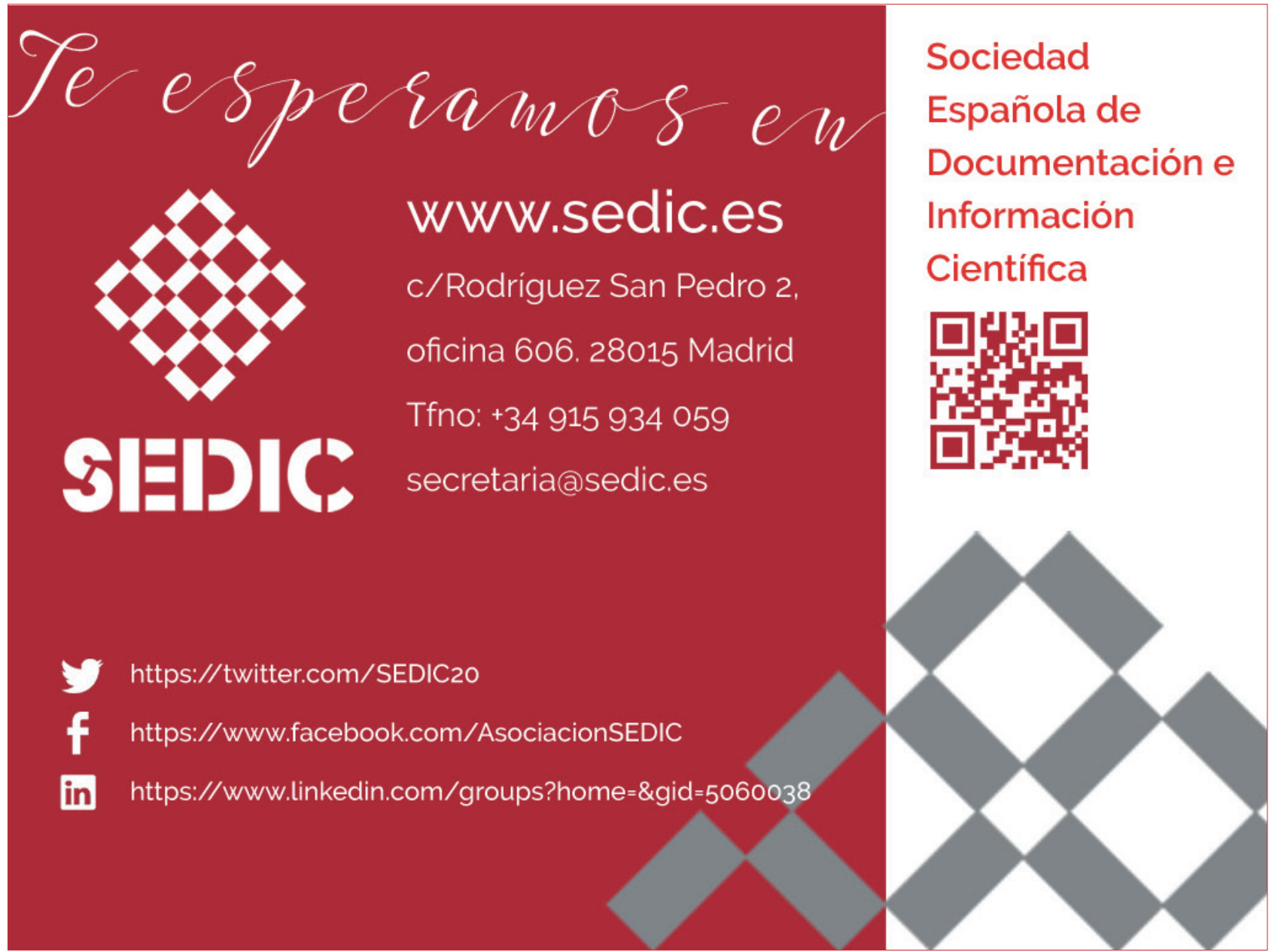

